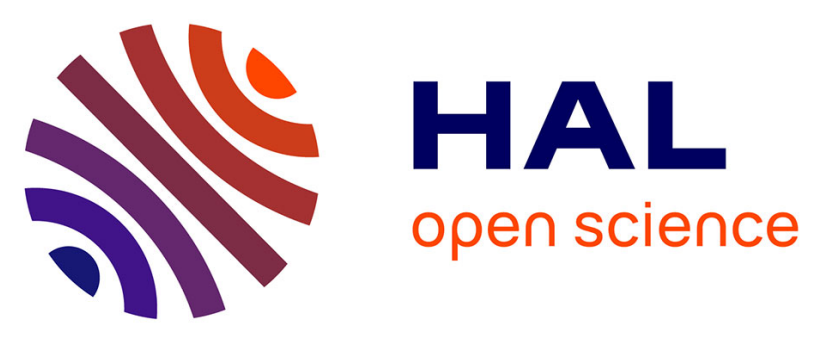

\title{
Towards low-power near-infrared modulators operating at telecom wavelengths: when graphene plasmons frustrate their metallic counterparts
}

Thomas Wood, Malik Kemiche, Jérémy Lhuillier, Pierre Demongodin, Bertrand Vilquin, Pédro Rojo Romeo, Aziz Benamrouche, Philippe Regreny, Ségolène Callard, Xavier Letartre, et al.

\section{To cite this version:}

Thomas Wood, Malik Kemiche, Jérémy Lhuillier, Pierre Demongodin, Bertrand Vilquin, et al.. Towards low-power near-infrared modulators operating at telecom wavelengths: when graphene plasmons frustrate their metallic counterparts. Journal of the Optical Society of America B, 2020, 37 (5), pp.1563. 10.1364/JOSAB.391277 . hal-02618631

\section{HAL Id: hal-02618631 \\ https://hal.science/hal-02618631}

Submitted on 8 Oct 2020

HAL is a multi-disciplinary open access archive for the deposit and dissemination of scientific research documents, whether they are published or not. The documents may come from teaching and research institutions in France or abroad, or from public or private research centers.
L'archive ouverte pluridisciplinaire HAL, est destinée au dépôt et à la diffusion de documents scientifiques de niveau recherche, publiés ou non, émanant des établissements d'enseignement et de recherche français ou étrangers, des laboratoires publics ou privés. 


\title{
Towards low-power near-infrared modulators operating at telecom wavelengths: when graphene plasmons frustrate their metallic counterparts
}

\author{
Thomas Wood, ${ }^{*}$ (i) Malik Kemiche, Jérémy Lhuillier, Pierre Demongodin, \\ Bertrand Vilquin, Pedro Rojo-Romeo, Aziz Benamrouche, Philippe Régrény, \\ Ségolène Callard, Xavier Letartre, and Christelle Monat \\ Ecole Centrale de Lyon, 36 Avenue Guy de Collongue, 69134, Ecully, France \\ ${ }^{*}$ Corresponding author: tomwood321@hotmail.com
}

Received 21 February 2020; revised 28 March 2020; accepted 31 March 2020; posted 31 March 2020 (Doc. ID 391277); published 1 May 2020

\begin{abstract}
A free-space electro-optic modulator device exploiting graphene's surface plasmon polariton (SPP) at near-infrared frequencies is proposed and theoretically studied. The device is made up of two resonant structures, the first being a metallic SPP displaying broadband absorption, and the second graphene's own SPP, which is shown to frustrate the metallic plasmon when excited, leading to a narrow reflectance peak. Doping of the graphene to achieve Fermi-level tuning is shown to shift the wavelength of the frustration phenomenon, thereby enabling the use of the device as a modulator. A reduction of $20 \%$ in the switching energy is expected due to the unique principle of operation which, crucially and contrary to most work in this field, does not rely on electroabsorption but electrorefraction changes in graphene. This coupled SPP resonator geometry also permits efficient channeling of optical energy from free space into graphene's SPP at near-infrared frequencies. (2020 Optical Society of America
\end{abstract}

\section{INTRODUCTION}

Since the reliable production of graphene monolayers by exfoliation in 2004, attention has turned to integrating graphene into on-chip optoelectronic circuits for data-communications applications [1]. The use of graphene to implement variableloss channels in electro-optical modulators in particular has been extensively investigated in the last decade [2-4]. Indeed, graphene's optical properties (absorption in particular) are tunable by changing its Fermi level via electrical gating. In the microwave region, where intraband electronic transitions dominate in graphene, a simple structure featuring parallel plate graphene electrodes has been shown to allow for up to $45 \%$ absorption of optical energy incident from free space, depending on the potential difference applied [5]. At higher optical frequencies - from the terahertz to optical domainsgraphene's absorption is usually dominated by interband electronic transitions, which, in a free-space, single-pass configuration, lead to a much weaker $2.3 \%$ absorption. The strongest light-graphene interactions occur when photons are confined in the vicinity of the carbon monolayer over long propagation distances, as through the use of linear waveguides [6], or for extended periods of time through the use of resonant structures [7]. Typical resonant strategies consist of inserting graphene within one-dimensional (1D) cavities made of dielectric or metal-dielectric thin-films stacks [8]. Further improvements may be made through the use of lateral structuration of the graphene's (dielectric or metallic) host medium to form Mie or plasmonic resonators, or through using photonic crystal cavities [9]. In such cases, absorption in graphene can reach above $80 \%$ and even $100 \%$, in the so-called perfect absorption regime $[10,11]$. The full width at-half maximum (FWHM) of the resonances at which near-total absorption occurs ranges from $5-20 \mathrm{~nm}$, while the center wavelength is determined by the fixed host medium geometry. Modulation in such devices can be achieved through electroabsorption changes in graphene, but this requires significant Fermi-level shifts of at least $200 \mathrm{meV}$ at room temperature due to thermal spreading of the onset of the interband transitions.

Another class of graphene-based modulators exploits graphene surface plasmon polaritons (SPPs), optical modes associated with periodic charge waves hosted on the graphene sheet due to the presence of highly mobile electrons. As for any optical mode, graphene's SPP is described with a modal index for which the real part describes the wavelength compression 
with respect to free-space radiation, and the imaginary part translates to the propagation losses. In the case of graphene's SPP, both parts of the modal index are typically much higher than for a "classical" metallic SPP and are strongly dependent on the graphene Fermi level. The resulting large momentum mismatch between light in free space and that in the SPP wave, and the tight confinement of optical energy above and below the plane that this entails, has hindered the efficient excitation of graphene's SPP at visible and near-infrared (NIR) frequencies to date. By contrast, in the mid-infrared (MIR) and far-infrared spectral regions, graphene's SPP wavelength takes values in excess of $100 \mathrm{~nm}$ at moderate doping levels, thereby allowing for the realization of practical devices using standard lithography techniques. In order to assure phase matching, either the graphene or its surroundings must be structured such that its spatial period is equal to the wavelength of the SPP (or a low integer multiple thereof) at the optical frequency of interest. The dependence of the SPP modal index on the graphene Fermi level then allows for an electrical control of the phase-matching frequency, forming the basis of operation of SPP-based electrooptical modulators. The high imaginary part of the modal index for graphene's SPP allows for efficient extinction of coupled optical energy, allowing for compact modulator devices. Coupling to graphene's SPP by structuring the graphene itself into strips, discs, or undulating forms has been demonstrated with device architectures in the MIR being proposed [12-14] and experimentally realized [15-17], including those making use of supplementary resonant structures to concentrate optical energy onto the graphene. Alternatively, using continuous graphene, and therefore advantageously facilitating homogeneous doping through electrical gating of the entire sheet, structuring of the local dielectric environment has been reported $[18,19]$ and experimentally realized [20] in the MIR domain. However, the high level of losses induced by graphene's SPP contributes to a significant spreading of the spectral response of the devices, with FWHM of the resonant extinction peaks of at least $100 \mathrm{~nm}$ in the MIR spectral region, corresponding to quality factors of between 10 and 100 . In modulator devices, this high FWHM translates again into the need for large Fermilevel displacements to switch between on and off states with a substantial extinction ratio.

The aforementioned graphene-based electro-optical modulators typically require large Fermi-level shifts, i.e., significant charge injection, to switch between the on and off states, thus increasing the device power consumption. To overcome this, we present a new SPP-based device architecture that exploits electrorefraction changes of graphene rather than electroabsorption ones. It is based on two complementary SPP resonators, the first being a conventional metallic SPP, and the second provided by graphene's SPP. We use the metallic SPP to concentrate energy onto the graphene sheet so that it acts as a pathway for photons to and from the graphene from free space, while also providing a spectrally broad absorption dip in the reflectance spectrum. When the graphene SPP resonance is spectrally superimposed and the exchange of optical energy between the two resonators is properly optimized, the metallic SPP absorption is partially frustrated, leading to the appearance of a narrow reflectance peak. We show that the spectral position of this peak is determined by the real part of graphene's SPP modal index, and that its formation is independent of losses induced by the graphene. As such, it is spectrally narrow-approximately $20 \mathrm{~nm} \mathrm{FWHM-and}$ may be tuned to the desired wavelength with minimal changes to the Fermi level. The underlying mechanism stands in contrast to previous graphene SPP-based modulators that typically exploit the tunable electroabsorption changes in graphene. In this paper, we evaluate the performance of the system as an electro-optical modulator using both coupled-mode theory and finite-difference time-domain simulations, and from an electrical perspective with a view to estimating its power consumption. We also discuss how this device concept can be implemented to be made compatible with NIR telecom wavelength signals. Finally, we underline that this coupled SPP resonator geometry also permits efficient channeling of optical energy from free space into graphene SPP at NIR frequencies, which has been challenging to achieve to date.

\section{COUPLED-MODE THEORY ANALYSIS}

We first use coupled mode theory (CMT) to show the principle of operation of the coupled resonator system, consisting of (a) a metallic SPP resonance and (b) graphene's SPP, without taking into account the detailed physical geometries. The response of each optical resonator is represented via the complex amplitude of the electromagnetic field it stores, while "ports" connect it to its neighboring environment as well as to an intrinsic loss channel, the latter representing absorption (or "ohmic") losses in the case of SPP resonances. This coupled resonator system is shown in Fig. 1(a). It is first composed of a perfect metal reflector representing the off-resonance response of the system whose reflectance is modified by the excitation of the metallic SPP centered on a frequency $\omega_{a}$ (or equivalently $\lambda_{a}$ ). In the metal SPP, photons have a decay rate in time of $1 / \tau_{a}$, the latter being directly proportional to the absorption losses. The metal SPP interacts with incoming $S_{+}$and outgoing $S_{-}$plane waves, representing the excitation source and the signal reflected back to free space, respectively, for which the coupling rate to the metal SPP is $1 / \tau_{c}$. The metal SPP (resonator $a$ ) also interacts with graphene's SPP (resonator $b$ ) centered on frequency $\omega_{b}$ (or equivalently $\lambda_{b}$ ), possessing an intrinsic absorption loss channel with a decay rate of $1 / \tau_{b}$, through a coupling whose strength is described by a coefficient K. We note that graphene's SPP cannot interact directly with optical energy from free space.

Rate equations Eqs. (1) and (2) pertaining to resonators $a$ and $b$, respectively, describe the time-resolved evolution of the complex amplitudes of the fields in each SPP resonance, taking into account the various loss and excitation channels,

$$
\begin{gathered}
\frac{d a}{d t}=\left[i \omega_{a}-\left(\frac{1}{\tau_{a}}+\frac{1}{\tau_{c}}\right)\right] a+i K b+\sqrt{\frac{2}{\tau_{c}}} S_{+}, \\
\frac{d b}{d t}=\left[i \omega_{b}-\frac{1}{\tau_{b}}\right] b+i K a .
\end{gathered}
$$

Admitting an imposed harmonic excitation with arbitrary pulsation $\omega$, Eqs. (1) and (2) become

$$
i\left[\left(\omega-\omega_{a}\right)-i\left(\frac{1}{\tau_{a}}+\frac{1}{\tau_{c}}\right)\right] a-i K b=\sqrt{\frac{2}{\tau_{c}}} S_{+},
$$


(a)
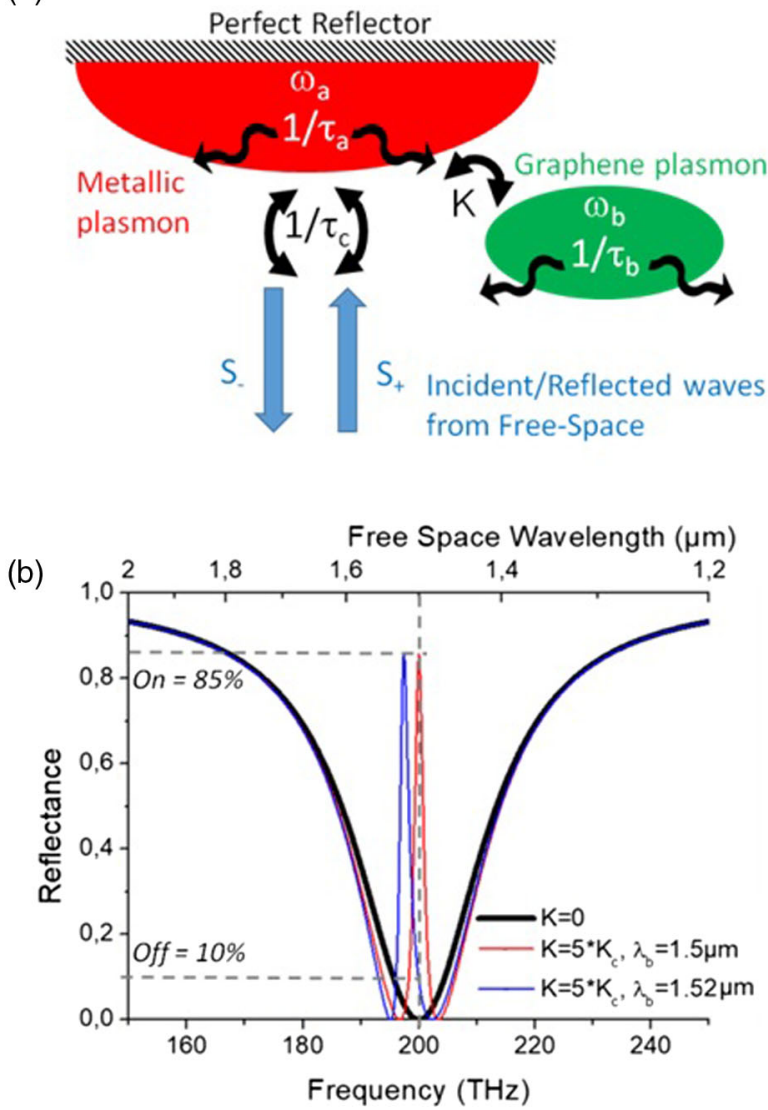

(d)

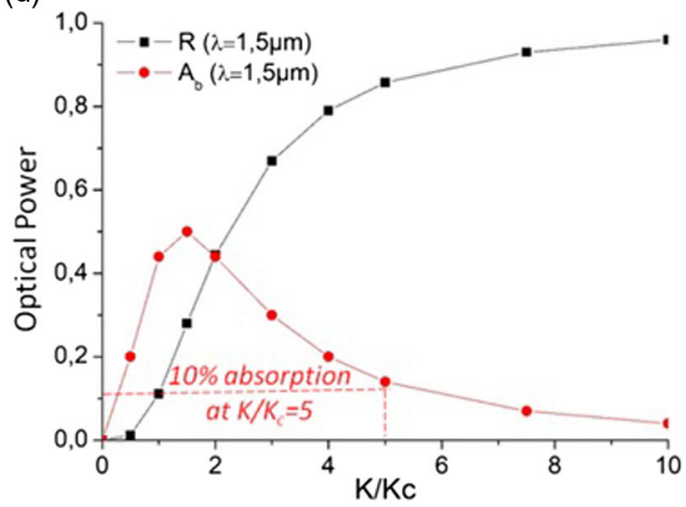

(c)

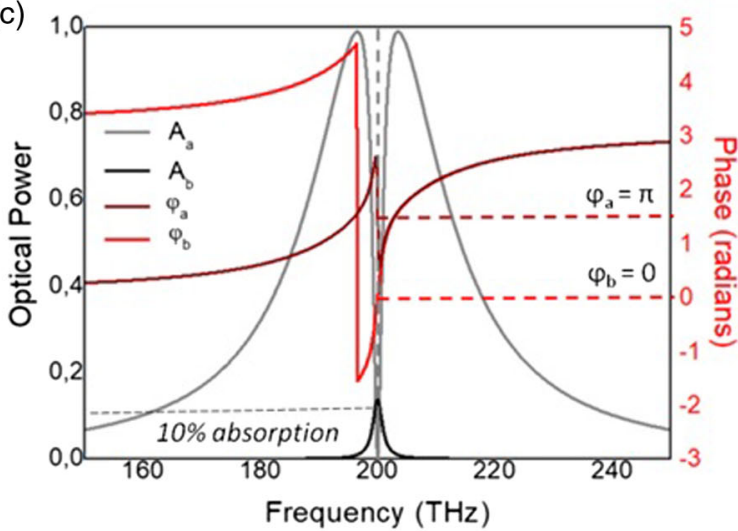

Fig. 1. (a) Schematic representation of the overall coupler device, showing the reservoirs representing the metal and graphene SPPs, and the associated exchanges, including with free space; (b) simulated reflectance spectra for $\lambda_{a}=1.5 \mu \mathrm{m}$, and various resonant wavelengths of graphene's SPP and coupling constants K; (c) normalized optical power absorbed in both SPPs $A_{a}$ and $A_{b}$ normalized to the incident intensity, and phase of SPPs with respect to the incident wave from free space for $K=5 * K_{c}, \lambda_{b}=1.5 \mu \mathrm{m}$; (d) normalized optical power reflected from the system and absorbed by graphene's SPP as a function of the coupling K between the metal and graphene SPPs for $\lambda_{a, b}=1.5 \mu \mathrm{m}$.

$$
i\left[\left(\omega-\omega_{b}\right)-i\left(\frac{1}{\tau_{b}}\right)\right] b-i K a=0 .
$$

These new equations can then be separated into distinct expressions for the amplitude of the optical fields in each resonator in the steady state, Eqs. (5) and (6), corresponding to resonators $a$ and $b$, respectively,

$$
\begin{gathered}
a=\frac{\sqrt{2 / \tau_{c}} S_{+}}{i\left[\left(\omega-\omega_{a}\right)-\frac{K^{2}}{\left(\omega-\omega_{b}\right)-i / \tau_{b}}\right]+\left(\frac{1}{\tau_{a}}+\frac{1}{\tau_{c}}\right)}, \\
b=\frac{K a}{\left(\omega-\omega_{b}\right)-i / \tau_{b}} .
\end{gathered}
$$

Finally, linking the optical energy stored in the resonators to the exchanges with free space, we have Eq. (7) describing the reflected field from the global system, with a reflection coefficient represented by Eq. (8),

$$
S_{-}=-S_{+}+\sqrt{\frac{2}{\tau_{c}}} a,
$$

$$
R=\left|\frac{S_{-}}{S_{+}}\right|^{2} .
$$

The normalized power A absorbed by each resonator (for a unitary incident field $S_{+}$) is given by Eq. (9):

$$
A_{a, b}=\frac{2|a, b|^{2}}{\tau_{a, b}} .
$$

We note that the only unknown parameter remaining in the system of CMT equations [Eqs. (6)-(9)] is now K, for which we define a "critical" value $\left.K_{c}=1 / \sqrt{(} \tau_{a} * \tau_{b}\right)$ as the mean absorption rate for the two SPPs, such that when $K>2 * K_{c}$ more power is exchanged between the two SPP resonators than is dissipated through absorption losses. Furthermore, we select $\tau_{a}$ equal to $\tau_{c}$ to obtain perfect absorption in the metallic SPP at its center frequency, in the absence of coupling to graphene's SPP $(K=0)$. The value chosen for $\tau_{a}=\tau_{c}=150 \mathrm{fs}$ yields an FWHM of the absorption dip of around $200 \mathrm{~nm} . \tau_{b}$ is 2 orders of magnitude higher at $15 \mathrm{ps}$, representing a lightly damped graphene SPP (for which the required doping level will be discussed later). We consider $\tau_{b}$ to be constant, while its dispersion is neglected given the minor shifts in the center wavelength of the graphene SPP resonance exploited below. We note that since 
$\tau_{a}=\tau_{c}<\tau_{b}$, many photons coupled into resonators $a$ and $b$ are subsequently radiated back to free space.

Figure 1(b) shows the overall reflectance of the coupled resonator system for a fixed center wavelength for the metallic SPP of $\lambda_{a}=1.5 \mu \mathrm{m}$, with various values taken by the graphene SPP center wavelength $\lambda_{b}$ and the coupling constant K. Figure 1(c) shows the normalized power absorbed in each resonator and the phase of the SPPs with respect to that of the incident wave for the supercritical coupling case $K=5 * K_{c}$ and $\lambda_{b}=1.5 \mu \mathrm{m}$. Under these conditions, no power is absorbed by the metallic SPP at $1.5 \mu \mathrm{m}$, and only around $10 \%$ of the incident power is absorbed by graphene's SPP. We note also from Fig. 1(c) that the phases of both resonators are opposite (difference of $\pi$ ) at $\lambda_{b}=1.5 \mu \mathrm{m}$; this is the principle of "frustration" of absorption in the metallic SPP resonance caused by destructive interference, and it leads to the formation of a narrow (FWHM $<20 \mathrm{~nm}$ ) reflectance peak corresponding to the relatively long lifetime $\tau_{b}$ of the graphene SPP. Given that absorption in resonators $a$ and $b$ are the only loss channels, we note that $A_{a}+A_{b}=1-R$, and hence that frustration occurs efficiently when $R>0.5$ (i.e., more power is reflected than absorbed). As can be seen in Fig. 1(d), showing the reflectance and absorption in graphene's SPP as a function of the coupling between resonators $a$ and $b$ when the center wavelengths are identical $\left(\lambda_{a, b}=1.5 \mu \mathrm{m}\right)$, the frustration mechanism is only efficient $(R>0.5)$ when the power exchanged between $a$ and $b$ is greater than their combined losses, i.e., for supercritical coupling, $K>2 * K_{c}$, It can also be seen that the maximum power absorbed in graphene's SPP is half of the incident power, which occurs for subcritical coupling $\left(K \sim 1.5 * K_{c}\right)$.

Modifying the Fermi level of graphene changes the modal index of graphene's SPP; hence the wavelength $\lambda_{b}$ (as described in Section 4.B below), with the consequence that the frequency at which the metallic SPP absorption is frustrated, i.e., the narrow reflection peak, is also displaced. This allows the modulation of reflected intensity at a given frequency value through graphene Fermi-level tuning. This is shown in Fig. 1(b), where the graphene SPP center wavelength $\lambda_{b}$ takes two values: 1.5 and $1.52 \mu \mathrm{m}$ (i.e., a shift equal to the FWHM of the resonance), yielding modulation of the reflectance at a free-space wavelength of $1.5 \mu \mathrm{m}$ between $10 \%$ ("off" state) and $85 \%$ ("on" state). We note that larger center wavelength shifts of around $40 \mathrm{~nm}$ for graphene's SPP could yield $0 \%$ reflectance in the off state, while the remaining $10 \%$ of optical power not reflected in the on state is absorbed by graphene's SPP [as visible in Figs. 1(c) and 1(d)].

We underline that graphene's SPP is an ideal candidate for resonator $b$, as it presents an electrically tunable modal index allowing us to modify the optical frequency at which light can be coupled to it (thereby shifting $\lambda_{b}$ ). In the following section, we present the implementation of such a system using two coupled SPP resonators. We start with the conditions of existence and modal index for graphene's SPP, followed by the design optimization of the graphene and metallic SPP resonator geometries. We then discuss the performance and feasibility of such a modulator device through highlighting some potential solutions to overcome the challenges associated with its operation in the NIR domain.

\section{IMPLEMENTATION OF THE COUPLED RESONATOR SYSTEM EXPLOITING GRAPHENE'S SPP}

\section{A. Dispersion of Graphene's SPP}

Graphene's surface conductivity, relating the surface current density to an external electric field, is defined as a function of optical frequency by the well-known Kubo function. The latter can be decomposed into (1) an intraband component at low frequencies, describing the high mobility of electrons and holes within the conduction and valence bands, similar to the so-called "Drude tail" for metals and (2) interband effects whose appearance is marked by a Fermi-Dirac-like step when the photon energy increases past twice the chemical potential $\mu$. Photons with frequencies higher than the "gap frequency" $\omega_{\text {gap }}=2 \mu / \hbar$ can cause interband electronic transitions and engender high optical absorption.

Figure 2 shows the different contributions to the overall surface conductivity from the inter- and intraband transitions. We admit fixed values of temperature and graphene's intrinsic hopping parameter, the latter describing the ease with which electrons may switch between adjacent potential wells on the graphene sheet, which is accepted as being around $2.7 \mathrm{eV}$ in the literature [21]. The plot in Fig. 2(d) shows that at high frequencies above an apparent threshold value $\omega_{\mathrm{th}}$, the real part of the surface conductivity takes positive (nonzero) values and the imaginary part negative values, equivalent to the dispersion observed in classical dielectric materials; in this spectral region we speak of "dielectric graphene." At frequencies below the threshold, we obtain a positive imaginary component and a smaller positive real one, resembling the optical properties of a metal; we therefore use the term "metallic graphene." Unsurprisingly, if graphene's SPP is to be accessible at a given optical frequency $\omega$, the graphene must be in a "metallic" state, implying that the threshold frequency $\omega_{\text {th }}$ must be higher than that used for excitation, $\omega_{t h}>\omega$. Given that the threshold frequency is always lower than $\omega_{\text {gap }}$, this condition requires a minimum value for the chemical potential, which increases the more one wishes to push the metallic behavior towards higher frequencies, associated with NIR and visible optical signals. A plot of the threshold frequency $\omega_{\text {th }}$ as a function of the chemical potential is shown in Fig. 2(e).

The dispersion relation for graphene's SPP is approximated in the nonretarded regime [22] by Eq. (10), in which the SPP modal index $N_{\text {spp }}$ at an angular frequency $\omega$ is shown to simply depend on the frequency-resolved surface conductivity $\sigma$ and dielectric constants of the surrounding materials $\varepsilon_{1,2} . k_{0}$ is the free-space wave vector and $\varepsilon_{0}$ the vacuum permittivity,

$$
N_{\mathrm{spp},(1 / \mathrm{GR} / 2)}=\left[\varepsilon_{0}\left(\varepsilon_{1}+\varepsilon_{2}\right) \frac{i \omega}{\sigma}\right] / k_{0} .
$$

Therefore, as discussed previously, once the immediate environment of the graphene is chosen, the most effective way to tune the effective index of the graphene SPP modal index is the chemical potential, which directly impacts the graphene surface conductivity $\sigma$ in Eq. (10). Figures 3(a)-3(c) show plots of the SPP's wavelength, skin depth (decay rate of energy above and below the graphene sheet), and decay length, three pertinent physical values directly linked to the complex modal index, 

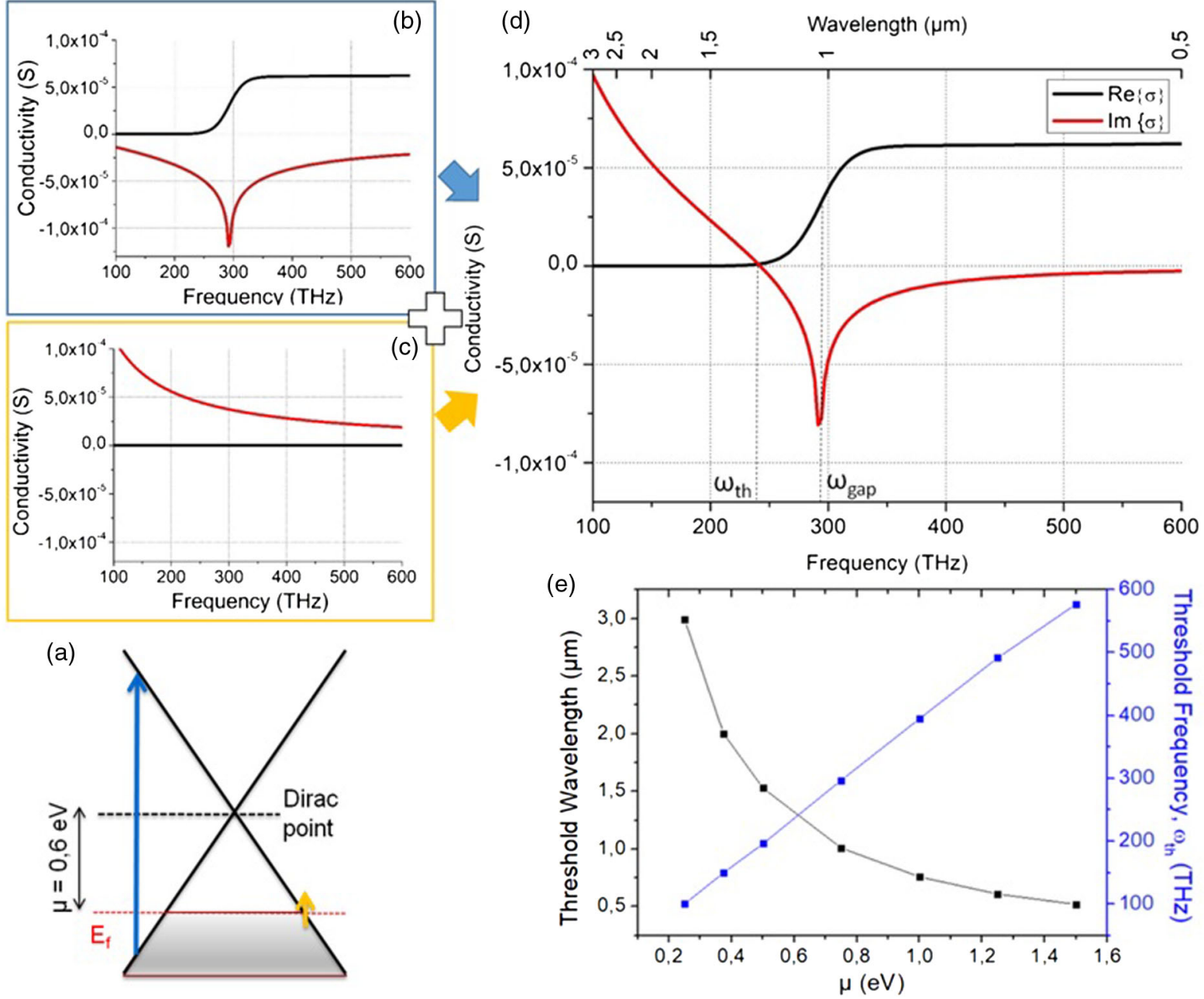

Fig. 2. Frequency dependence of graphene's surface conductivity for a temperature of $300 \mathrm{~K}$, value for the tight-binding hopping parameter of $2.7 \mathrm{eV}$, and a chemical potential (i.e., separation of Fermi level $E_{f}$ from Dirac point) $\mu=0.6 \mathrm{eV}$, the latter chosen as an example to yield metallic graphene at NIR frequencies. (a) Representation of band diagram with corresponding electronic transitions; (b) and (c) contribution to the surface conductivity of the inter- and intraband transitions; (d) total surface conductivity showing the gap frequency $\omega_{\text {gap }}$ and dielectric-metallic threshold frequency $\omega_{\text {th }}$; (e) threshold frequency and free-space wavelength for metallic graphene as a function of the graphene Fermi-level shift, $\mu$.
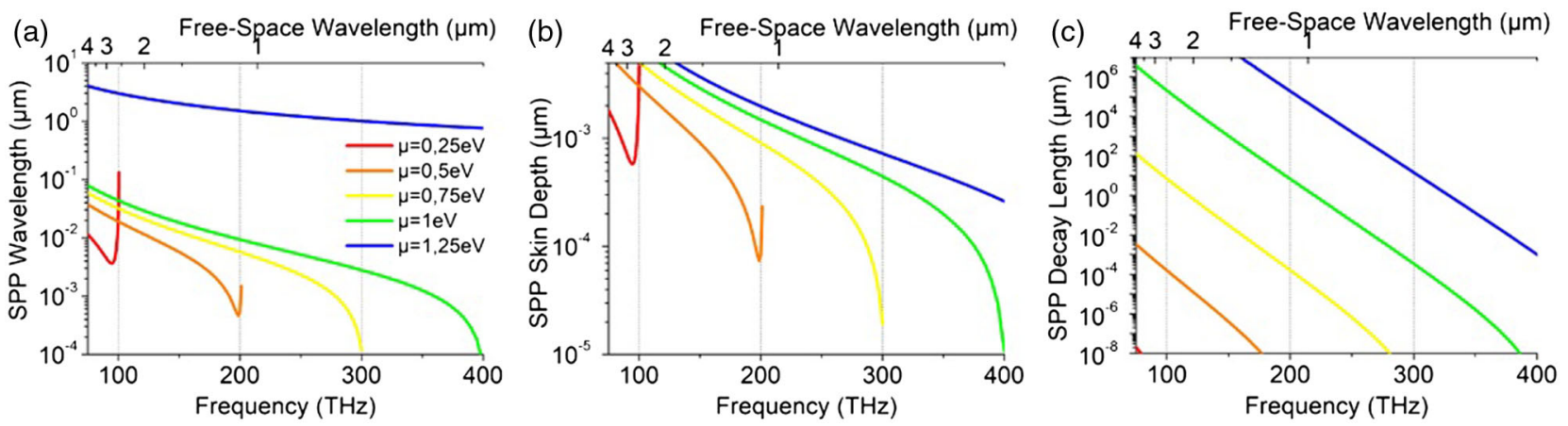

Fig. 3. Frequency-resolved plots for different parameters of graphene's SPP. (a) Wavelength; (b) skin depth (representing decay on either side of the graphene plane); (c) decay length, for various chemical potentials and $\varepsilon_{1}=\varepsilon_{2}=2.25$.

as functions of frequency for $\varepsilon_{1}=\varepsilon_{2}=2.25$. This dielectric environment represents graphene sandwiched in silica or some similar medium, of refractive index $n=1.5$, in accordance with the modulator structure proposed later in this paper. It can be seen that the SPP wavelength tends to zero (the real part of the modal index tends to infinity) as the frequency approaches the threshold value $\omega_{\text {th }}$. For instance, at a free-space wavelength of $1.5 \mu \mathrm{m}$ and for a doping level of $\mu=1 \mathrm{eV}$, graphene's SPP has a 
wavelength of the order of $10 \mathrm{~nm}$ and a skin depth of the order of $1 \mathrm{~nm}$. These values directly impact the required dimensions of the grating structures needed to couple light to graphene SPPs. Indeed, to realize the coupled resonator system of Section $2, \lambda_{b}$ is determined by the phase-matching condition under which photons can be channeled to the graphene SPP, as will be discussed in the following section. We also note that the SPP decay length shown in Fig. 3(c) is proportional to the photon lifetime in the graphene SPP, i.e., $\tau_{b}$ in the CMT model of Section 2. At a fixed optical frequency, the approximate change in decay length for a change in $100 \mathrm{meV}$ of graphene's chemical potential can be estimated as spanning one decade. In light of the small chemical potential shifts needed to shift the frustration peak by its FWHM and thereby switch the modulator's state, which are shown later to be around $20 \mathrm{meV}$, these modest variations of graphene SPP loss are consistent with the fixed $\tau_{b}$ assumption in the CMT model.

\section{B. Description of the Coupled SPP Resonator System}

The resonator $a$, i.e., the metallic SPP coupled to free-space light, is first implemented by a classic metal/semiconductor grating structure, in this case $\mathrm{Au} / \mathrm{Si}$, for which the grating is formed in the high-index semiconductor that is separated from the metallic substrate by a low-index slot region (for example, silica) of $20 \mathrm{~nm}$ thickness into which graphene can be inserted, as depicted in Fig. 4(a). A correct selection of the Si grating period and depth allows us to tailor the formation of the metallic SPP to any frequency in the infrared spectral region, as presented in the following section. Furthermore, as shown in Fig. 4(b), giving a representation of the distribution of the electric field for the different modes of interest, the low-index slot region hosts a significant enhancement in the field intensity on the graphene due to the conservation of the electric displacement vector component oriented across the slot. This enhancement is key to ensuring a high rate of photon exchange between the metallic

(a) Geometric parameters

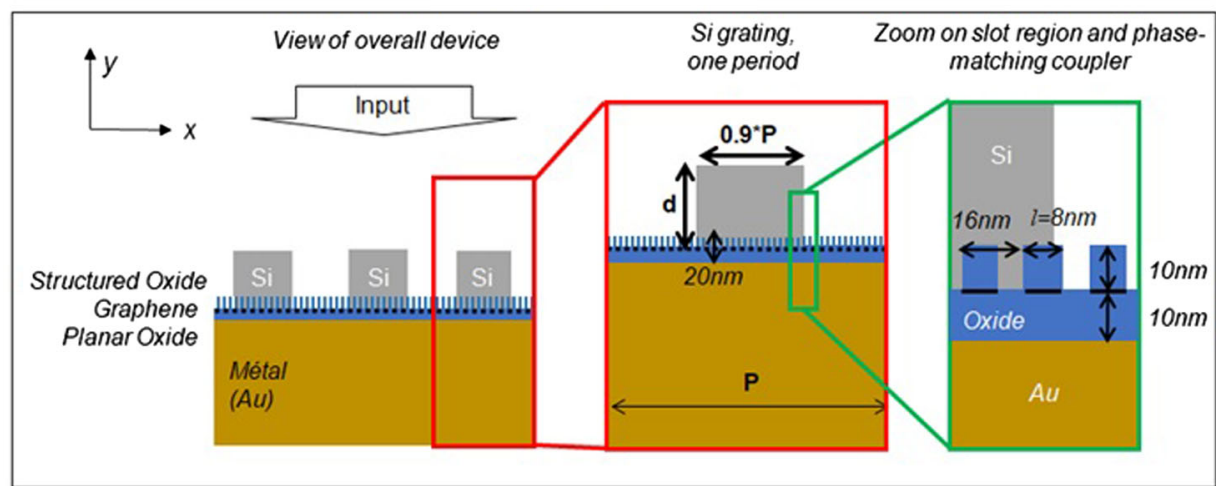

(b) Fields in coupler device

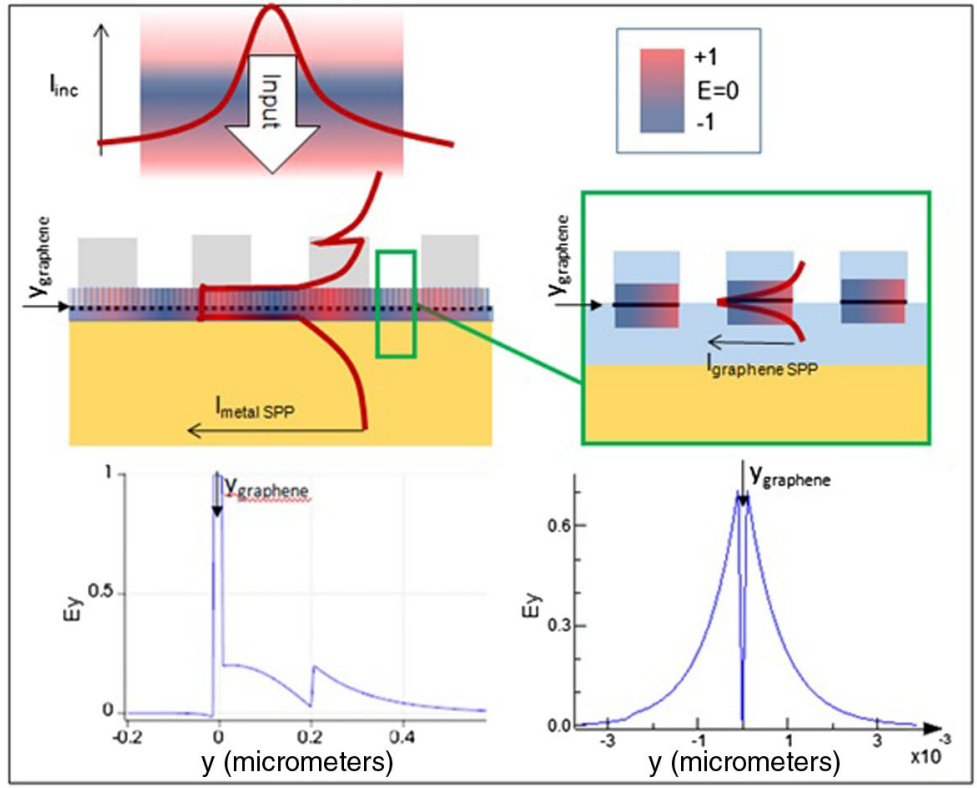

Fig. 4. (a) Overall view of the device structure to excite the graphene SPP, zoom on one period of the metal/semiconductor (Au/Si) coupler, and the oxide-filled slot region sandwiching the graphene (black lines), structured to form the phase-matching coupler; (b) representations of electric-field amplitude and intensity distributions in the different stages of the coupler device for the metallic SPP (thickness of Si teeth in this example $=200 \mathrm{~nm}$ ) and graphene's SPP, showing a strong spatial overlap of the $y$ component of the fields associated with both resonances in the slot region; $y=0$ is taken as the position of the graphene sheet, represented by the dashed black line. 
SPP and graphene's SPP, giving rise to supercritical values of the coupling constant, following the guidelines provided by the CMT analysis $\left(K>2 * K_{c}\right)$. The thickness of the slot is a compromise between being sufficiently narrow so as to host significant field enhancement, and wide enough so as to ensure that the evanescent field of the graphene SPP is primarily situated in the low-index material that the slot is filled with. Indeed, if the slot were too narrow, the tails of the evanescent fields would penetrate into the high-index $\mathrm{Si}$, and the modal index would be further increased, leading to higher losses and an even shorter SPP wavelength.

Having confined optical energy to the vicinity of the graphene, we now turn our attention to the problem of phase matching between light confined in the metal SPP and that which we seek to inject into graphene's SPP mode, a task significantly complicated by the exceptionally low wavelength of graphene's SPP for reasonable doping levels. As for the excitation of any guided or surface mode, phase matching can be achieved by structuring the host medium of the SPP wave with features possessing dimensions of the order of the SPP wavelength. In this case, the periodic structuration is performed on the oxide material composing the slot, within which the graphene layer is sandwiched, so as to form a square-tooth grating with a duty cycle of $50 \%$, as shown in Fig. 4 . A proper control of the structuration depth also allows for the graphene itself to be cut into ribbons, possessing a length $l$ equal to that of the square oxide teeth. In order to determine the required structure period, we first select a graphene Fermi level and use the dispersion curves of Fig. 3(a) to find the SPP wavelength, which must be equal to the square-tooth length (i.e., half of the period). We choose the highest chemical potential for which doping is feasibly achievable of $1 \mathrm{eV}$ (see Section 7.A). For operation at a free-space wavelength of $1.55 \mu \mathrm{m}$, we obtain a graphene SPP wavelength of $8 \mathrm{~nm}$, and so $16 \mathrm{~nm}$ is therefore selected as the period for the structuration. We note that this length scale is clearly incompatible with classic nanofabrication lithography methods, such as electron-beam or photolithography, but a self-organization technique is proposed in the experimental feasibility paragraphs (Section 7) at the end of the paper to obtain semiconductor surfaces featuring the fine structures required. Furthermore, Section 7 contains further simulations showing the expected device performance if the period of the phase-matching grating is dilated to near $50 \mathrm{~nm}$, thereby becoming compatible with electron-beam lithography fabrication.

\section{DESIGN OPTIMIZATION OF THE METALLIC/GRAPHENE SPP-COUPLED RESONATOR SYSTEM THROUGH RCWA AND 2D FDTD SIMULATIONS}

In order to determine the optimal dimensions for the Si grating coupler enabling the excitation of the metallic SPP, one period-representative of the entire device due to the periodic nature of the simulations-was studied using the rigorous coupled wave analysis (RCWA) technique. Fully dispersive dielectric constants were taken into account for the metal $(\mathrm{Au})$ and semiconductor $(\mathrm{Si})$ materials, and 30 plane waves were used in the Fourier series expansions. Parameter sweeps, shown in
Figs. 5(a) and 5(b), on the geometric variables of the Si grating, shown in Fig. 4(a), yielded the following values in order to minimize reflection via absorption in the fundamental metallic SPP resonance at $1.55 \mu \mathrm{m}$ wavelength (optical frequency $\sim 200 \mathrm{THz}$ ), $\mathrm{P}=1278 \mathrm{~nm}, \mathrm{~d}=70 \mathrm{~nm}$. Figure 5(c) shows the reflectance calculated with $2 \mathrm{D}$ FDTD for a device composed of a 10-period grating with these optimized parameters illuminated by a Gaussian beam from free space (waist $=5.5 \mu \mathrm{m}$ ) under normal incidence, using perfectly matched layer (PML) absorbing boundaries on all sides. In this case, the chemical potential of graphene is zero, prohibiting the presence of graphene's SPP, whose spectral signature is therefore absent. A comparison is provided with the RCWA simulated reflectance, for which the reflectance dip is narrower due to the single, normal-incidence plane wave used (in contrast to the Gaussian beam of the FDTD simulations). Figures 5(d) and 5(e) show the optical intensity on the graphene with respect to that of the incident beam over the width of the coupler and the average enhancement upon integrating over the two central periods of the Si grating, respectively. One can see that an average 30 -fold increase in the optical intensity is produced on the graphene near the fundamental mode of the metallic SPP at wavelength $1.55 \mu \mathrm{m}$.

In order to explore the tunability of the narrow reflectivity peak associated with the frustration of the absorption in the metallic SPP by graphene's SPP, the Fermi level of the graphene was varied from 0.92 to $1 \mathrm{eV}$ by steps of $400 \mathrm{meV}$, and the resulting reflection coefficients are shown in Fig. 6. As expected from the CMT analysis, a relatively narrow reflectance peak can be seen (see, for instance, the red curve for $\mu=0.96 \mathrm{eV}$ ) within the $100 \mathrm{~nm}$ broad reflectance dip around $1.55 \mu \mathrm{m}$ provided by the coupling to the metallic SPP.

For the lowest chemical potential of $0.92 \mathrm{eV}$, small perturbations to the reflectance spectrum are seen indicating injection of energy into graphene's SPP; however, the degree of frustration remains low. This is due to the fact that such interactions are either (1) off-resonance with respect to the metallic SPP (meaning a low concentration of optical energy in the slot and hence a low coupling constant $\mathrm{K}$ ), as is the case for the perturbations below $\lambda=1.4 \mu \mathrm{m}$ or (2) because of the high levels of loss in graphene's SPP due to proximity to the threshold frequency. Increasing the chemical potential blueshifts the frequencies at which injection into graphene's SPP occur due to the decrease in the modal index (or increase in the SPP wavelength) at a given optical frequency, thus blueshifting the frustration phenomenon. This can be visualized in the lower panel of Fig. 6; the lowest frequency excitation of graphene's SPP occurs for each chemical potential when the corresponding dispersion curve crosses the horizontal line representing the graphene ribbon length $l$. Subsequent coupling occurs for SPP wavelengths matching the high-order harmonics of the ribbon length (i.e., $2 l / 3, l / 2 \ldots$ ). Note that, in Fig. 6(b), the SPP wavelength has been linearly scaled by a factor of 0.82 across the entire frequency range considered, to take into account the reflection phase change of the SPP at the ribbon extremities. We note that the importance of the frustration phenomenon (i.e., the absolute intensity of the narrow peaks formed) increases with spectral proximity to the metallic SPP's center wavelength of $1.55 \mu \mathrm{m}$ due to increased energy confinement in the slot 
(a)

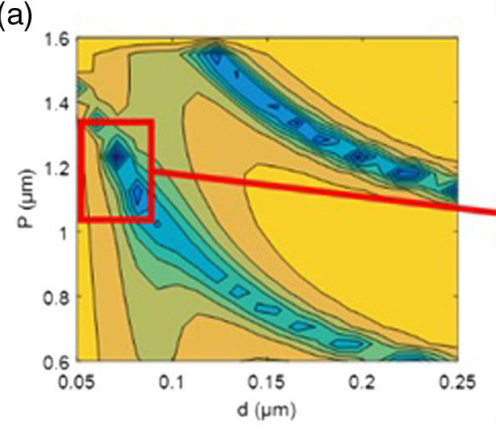

(d)

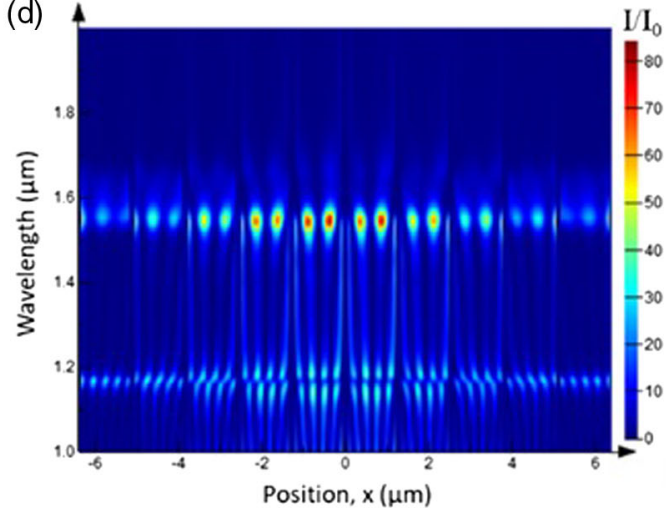

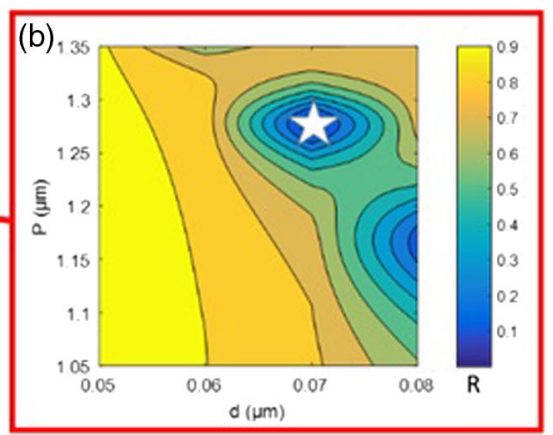

(c) 1.0

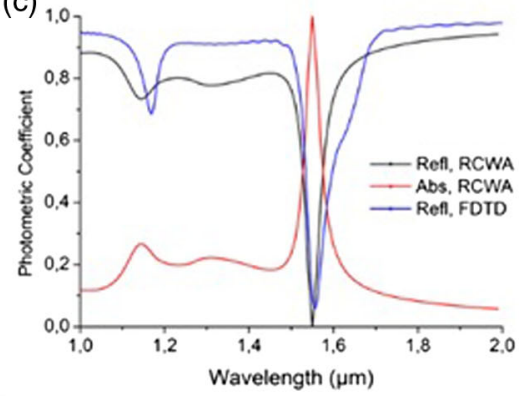

(e)

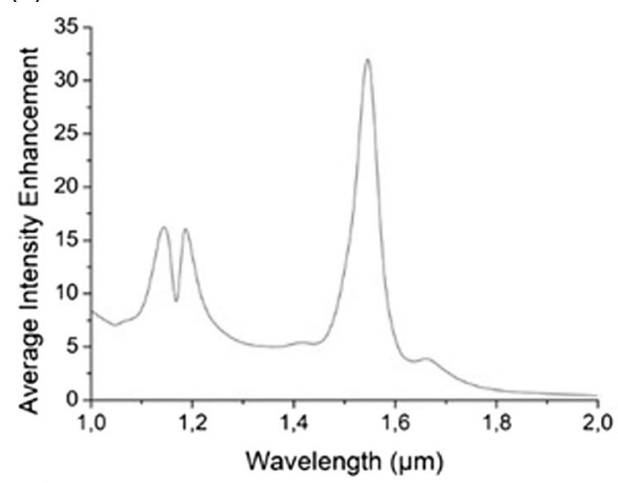

Fig. 5. (a) Reflectance at wavelength $1.55 \mu \mathrm{m}$ as a function of the Si grating period and depth; (b) zoom on indicated region of (a), allowing the optimum values (marked by the white star) to be deduced; (c) RCWA (reflection and absorption coefficients) and FDTD (reflection coefficient of a device composed of 10 periods) for the optimum grating parameters; (d) wavelength-resolved optical intensity enhancement at $y=y_{\text {graphene }}$ (indicated in Fig. 4) over the 10-period coupler width, x, obtained by FDTD simulations; (e) average optical intensity enhancement over the two central periods of the Si grating as a function of wavelength.

surrounding the graphene, leading to supercritical coupling between the two SPP resonators. Indeed, between 1.3-1.4 $\mu \mathrm{m}$, for example, perturbations of the base absorption set by the metallic SPP are much weaker due to the decrease in the coupling between the two SPP resonances away from the central resonance of the metallic SPP. This suggests a working spectral window for the device, i.e., the range of wavelengths at which the modulation contrast induced by frustration remains acceptable, of around $50 \mathrm{~nm}$, centered on $1.55 \mu \mathrm{m}$.

\section{EVALUATION OF THE SYSTEM PERFORMANCE AS A MODULATOR}

As an example of the device's performance as an electro-optic modulator, if one considers the reflectance near $1.55 \mu \mathrm{m}$, it can be seen in Fig. 6 that the reflectance can be tuned between over $80 \%$ (on state) and $6 \%$ (off state). The finesse of the reflectance peak allows for very small shifts of the chemical potential in order to switch between these on and off states. Further 2D FDTD simulations were carried out with steps between the Fermi levels of $10 \mathrm{meV}$, as shown in Fig. 7, and from these, the reflectance at a fixed wavelength as a function of the Fermi level can be obtained, demonstrating a near-linear relationship on $\mu$. Two examples of working points for the modulator device have been determined: (a) one maximizing the reflectance contrast, and (b) one demonstrating a reduced switching energy with respect to interband absorption-based modulators. In the case of (a), a chemical potential shift of $20 \mathrm{meV}$ around a central value of $1.01 \mathrm{eV}$ is required to switch the SPP-based modulator between $87 \%$ reflection in the on state and $6 \%$ reflection in the off state, i.e., a contrast $10 \cdot \log _{10}\left(R_{\min } / R_{\max }\right)$ corresponding to $\mathrm{a}-11.6 \mathrm{~dB}$ modulation. In case (b), a chemical potential shift of $10 \mathrm{meV}$ around a central value of $1.015 \mathrm{eV}$ is required to give a $-10 \mathrm{~dB}$ modulation (between an off state at $6 \%$ and an on state at $62 \%$ reflectance). For comparison, for a modulator based on variations of the interband absorption of graphene such as that in [9], a $200 \mathrm{meV}$ chemical potential variation spanning from approximately 0.3 to $0.5 \mathrm{eV}$ is required to modify the modulator's response over a $10 \mathrm{~dB}$ range. We conclude that a change in reflectance at a precise wavelength requires significantly greater shifts of the chemical potential for modulator architectures operating using changes in the interband absorption coefficient of graphene upon electrical gating. This is due to the broadening of the Fermi-Dirac-like step between absorbing and transparent behaviors due to temperature effects, partly dependent on the probing optical intensity, which are beyond the scope of the present article. In this work, it is the spectral finesse of the reflection peak generated by the frustration of the metallic SPP by graphene's SPP, and the high sensitivity of the phasematching conditions to the Fermi level, that allow surprisingly high degrees of modulation to be obtained for relatively small changes in the Fermi level.

To give a quantitative estimation of the modulator device's electrical performance, we use a simplified model based on 


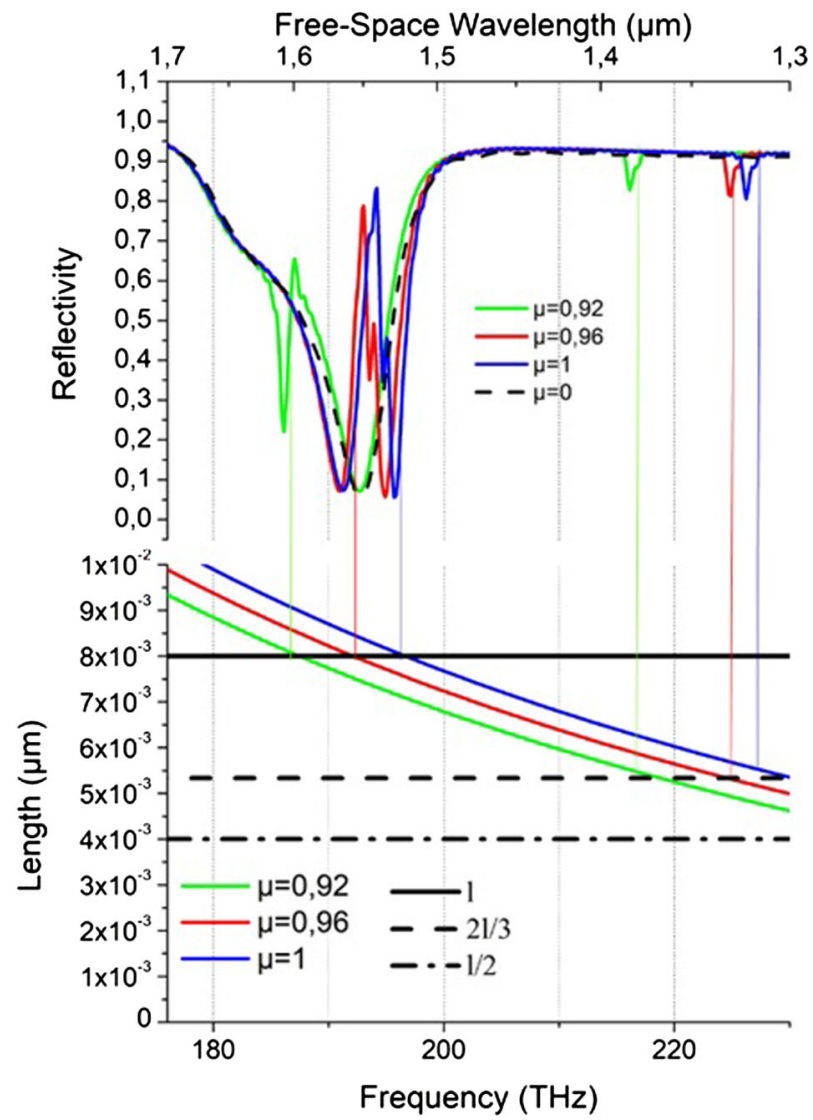

Fig. 6. Upper, spectrally resolved reflectance from the 10-period device; lower, dispersion of graphene SPP wavelength for different chemical potentials; also shown are the oxide-teeth length $(l)$ and harmonics thereof to highlight the frequencies at which graphene's SPP is excited through phase matching.

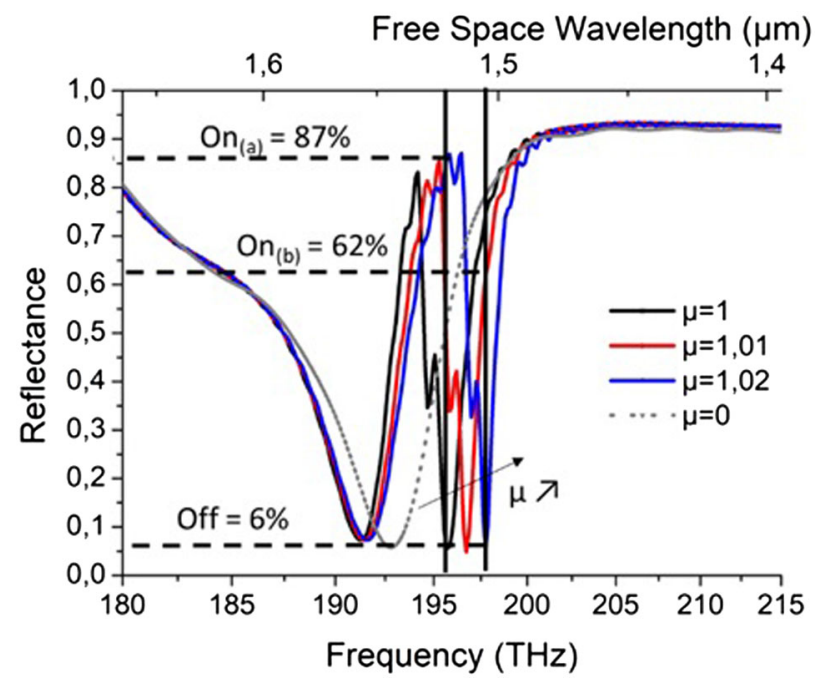

Fig. 7. Fine sweep of Fermi level between 1 and $1.02 \mathrm{eV}$ in FDTD, showing modulation between $6 \%$ and $87 \%$ near $1.55 \mu \mathrm{m}$; the lowest reflectance (off state) is $6 \%$, while two values are chosen for the on state, corresponding to (a) $87 \%$ giving the highest optical modulation contrast of $-11.6 \mathrm{~dB}$ and (b) $62 \%$ used in the switching energy calculation example. a planar capacitor of which the ensemble of graphene ribbons form one plate and the metallic substrate the other. For an active area of $10 * 10 \mu \mathrm{m}$, a capacitor thickness of $10 \mathrm{~nm}$ and supposing a DC relative dielectric constant of the dielectric spacer of 4 , the capacitance $\mathrm{C}$ is evaluated at $0.4 \mathrm{pF}$. The chemical potential of graphene can be approximated by the applied bias voltage (assuming that the graphene is undoped in the absence of an applied voltage) through the relation: $V=\left(\mu / \hbar \cdot v_{f}\right)^{2} \cdot\left(q_{e} \cdot d / \pi \cdot \varepsilon_{0} \cdot \varepsilon_{d}\right)$, where $\hbar$ is the reduced Planck constant, $v_{f}=1.5 \cdot 10^{6} \mathrm{~m} / \mathrm{s}$ is the Fermi velocity, $q_{e}$ is the charge of an electron, $d$ is the capacitor thickness, $\varepsilon_{0}$ is the permittivity of free space, and $\varepsilon_{d}$ is the DC relative dielectric constant of the dielectric spacer. Finally, in order to estimate the energy stored in the capacitor $E$, the following relation is used: $E=(1 / 2) C V^{2}$. The energy required to switch the modulator (energy per bit) is calculated by evaluating the difference in stored energy at the chemical potentials corresponding to the on and off states using the two previous equations, and corresponds to $6.4 \mathrm{pJ} / \mathrm{bit}$. The energy per bit calculated in the case of an interband absorption-based device with equivalent capacitance (and hence surface area) is $25 \%$ higher at $8.3 \mathrm{pJ} / \mathrm{bit}$, calculated using the bias voltages corresponding to chemical potentials of 0.3 and $0.5 \mathrm{eV}$, which correspond to transitioning between fully allowing or forbidding interband transitions for an operating temperature of $300 \mathrm{~K}$. Despite the marked improvement, the energy consumptions are of the same order of magnitude because the energy stored in the capacitor varies as $\mu^{4}$, and the higher average value of $\mu$ for the SPP-based device $(1.015 \mathrm{eV})$ with respect to an interband absorption-based device $(0.4 \mathrm{eV})$ operating in the NIR domain compensates for the significantly lower absolute change in $\mu$ for the SPP-based device upon switching. We also note that, as is detailed in Section 7.A, if static doping in the absence of a bias voltage can be achieved, the energy per bit can be reduced by an order of magnitude.

In order to provide a comparison with existing graphenebased technology in the literature, Ref. [23] details an electro-optic modulator addressed in normal incidence for which the variation of reflectance is caused by the transition between allowing and forbidding interband transitions. While the energy per bit is not directly quoted, the device dimensions are $20 \mu \mathrm{m} * 20 \mu \mathrm{m}$, the capacitance is cited as $6.8 \mathrm{pF}$, and the drive voltage swing (absolute values not given) for modulation is $2 \mathrm{~V}$, thereby leading to a calculable minimum energy consumption of $13.6 \mathrm{pJ} / \mathrm{bit}$. Comparison with waveguideintegrated modulators is more complex, due to the significantly smaller active cross sections employed (typically of the order of $0.1 \mu \mathrm{m}^{2}$ ) in integrated applications. If a linear relationship is supposed between active cross section and energy consumption, based on the energy per bit figure for Si-based waveguideintegrated modulators established in Ref. [24] of $1 \mathrm{fJ} / \mathrm{bit}$, an acceptable figure for a normal incidence addressed modulator of dimensions $10 \mu \mathrm{m} * 10 \mu \mathrm{m}$ would be of the order of $1 \mathrm{pJ} / \mathrm{bit}$, which is comparable to that estimated for our device of $6.4 \mathrm{pJ} / \mathrm{bit}$.

Finally, the maximum operating frequency can be calculated using the equation $f_{\max }=1 /(2 \pi R C)$, yielding $8 \mathrm{GHz}$, assuming a series resistance $R=50 \Omega$ (independent of the capacitor 
itself). We note that this frequency limitation is due to the electrical connection, rather than the intrinsic rate at which the SPP modulator can be switched.

\section{DISCUSSION}

Building on the work discussed in the introduction, we have presented a new device architecture for achieving electro-optical modulation using graphene in the NIR domain. The full spectral response of the proposed system over a window of $100 \mathrm{~nm}$ centered on $1.55 \mu \mathrm{m}$-in particular the position of a narrow reflectance peak-is modifiable electrically, and its tuning requires only small shifts in graphene's Fermi level. By sweeping the reflectance peak over a fixed operating frequency, we show that we can obtain a modulation contrast in the reflection configuration, i.e., $10 \cdot \log _{10}\left(R_{\min } / R_{\max }\right.$ ), of $-11.6 \mathrm{~dB}$ (corresponding to $6 \%$ reflectance in the off state and $87 \%$ in the on state).

As with many of the previously cited works, we make use of a coupled resonant system to enhance the potential optical coupling to graphene's SPP and hence improve the modulation contrast in a reflection configuration. Our work complements prior studies of the so-called coupled resonator induced transparency (CRIT) effect, which relies on the destructive interference of two spectrally overlapping resonators in order to create a spectrally tunable transparent window embedded within a broadband absorption region. Devices based on this operating principle have been proposed in the $5-10 \mu \mathrm{m}$ wavelength region $[12,19]$. Our approach differs from previous works in the CRIT field in a few key ways: (i) Both resonators are not free to exchange with free space (only the metal SPP may do so), whereas in other CRIT works both resonators have coupling structures to allow phase matching with free-space radiation. (ii) Contrary to the CRIT works cited, the spectral widths of the two resonators differ by an order of magnitude, and it is the narrow width of the graphene SPP resonance that allows for low switching energies.

We underline that the exploitation of the electrorefractive effect in graphene rather than electroabsorption (i.e., using electrical tuning of the real part of the modal index of graphene's SPP to selectively frustrate a coupled resonator, rather than seeking to directly absorb energy onto the graphene sheet) leads to improvements in the finesse of the spectral response of the system and so to reduced power consumption when used at a fixed wavelength as a modulator. Our device allows us to maintain resonant features in the reflectance spectra with quality factors $\left(\lambda_{\text {center }} / \mathrm{FWHM}\right)$ comparable to the state of the art $(Q \sim 75)$ while reducing the Fermi-level shift required for modulation $(\Delta \mu=20 \mathrm{meV})$ by an order of magnitude compared with that reported for interband absorption-based modulators operating in the NIR [9] or those relying on absorption of energy into "unaccompanied" graphene SPP resonances in the MIR [16] $(\Delta \mu=200 \mathrm{meV}$ for both references). In the case of CRIT approaches exploiting interference between dual graphene SPP resonances, we can extrapolate from the literature [19] that similar degrees of modulation contrast to those proposed in the present work can be achieved in a transmission configuration for $\Delta \mu=40 \mathrm{meV}$, albeit in the MIR spectral region.
We also note the potential for operation of our device at longer wavelengths, for example, in the MIR spectral region, which could relax the two major technological constraints addressed in Section 7, namely, the average doping level of graphene, and the period for the phase-matching coupler. While the architecture presented in this work was designed for operation in the NIR, this window can be readily shifted spectrally by redesigning the $\mathrm{Si}$ grating to displace the absorption peak of the metallic SPP and concentrate light onto the graphene sheet at higher wavelengths. We recall here that the doping level of graphene and the SPP wavelength (which we seek to match with the phase-matching coupler) are intrinsically linked, as shown in Fig. 3(a). At higher operating wavelengths, one could therefore choose to relax one, or to a lesser degree both, of these parameters, leading to lower chemical potentials of the graphene and higher periods for the phase-matching coupler, both of which would facilitate the device fabrication. We note also that the electrical consumption of a modulator device would be also reduced in the case of lower doping levels, as discussed in Section 5 .

Finally, we note that the coupled resonator system presented can be used in order to couple light durably into graphene's SPP (i.e., without it being subsequently decoupled to frustrate the metal SPP). Due to the high imaginary part of the SPP index and the relatively short photon lifetime implied, such a coupling manifests itself in the CMT modeling as an absorption of energy in resonator $b$. We see from Fig. 1(d) that for subcritical coupling $\left(K=1.5 * K_{c}\right)$, a maximum absorption in graphene's SPP of $50 \%$ of the coupled optical energy may be obtained for a correctly chosen device geometry. We remind the reader that CMT modeling is purely in the time domain and features no spatial component. In reality, energy coupled into graphene's SPP will propagate while undergoing linear attenuation, over a distance characterized by the decay length given in Fig. 3(c). In the case of the doping levels $(\sim 1 \mathrm{eV})$ and operating wavelength $(1.55 \mu \mathrm{m})$ presented here, we can see from Fig. 3(c) that the propagation length is approximately $10 \mu \mathrm{m}$, which is below the overall dimensions of the illuminated coupler device. Therefore, the CMT model can be considered valid over the spatial extent of the device, as a negligible quantity of energy coupled to graphene's SPP would be able to propagate out from this volume, and all coupled energy can be considered absorbed locally. However, working in the MIR spectral region, the propagation length of graphene's SPP can be increased by orders of magnitude, opening up possibilities for ultracompact optoelectronic devices that are compatible with current nanofabrication techniques.

\section{EXPERIMENTAL FEASIBILITY}

While the underlying physics behind the operation of our proposed device may be transposed to any operating wavelength, in particular — as suggested in the previous section— the MIR wavelength range in which fabrication constraints would be relaxed, we seek nevertheless to demonstrate the feasibility of operation at NIR telecom wavelengths. To this length, the following sections provide some technological solutions that can overcome the challenges associated with the fabrication of the component described previously for operation near the $1.55 \mu \mathrm{m}$ 
wavelength, namely, the doping of graphene to high chemical potentials and the fabrication of structured surfaces with periods of the order of $10 \mathrm{~nm}$.

We note that while the production of devices exploiting graphene's surface plasmon at telecommunication wavelengths remains challenging, demonstrations do exist in the literature. Namely, in Ref. [17] the authors report the observation of the dipolar mode of the localized surface plasmon resonance of graphene at a wavelength of $2 \mu \mathrm{m}$, thus very close to the telecommunications wavelengths targeted in our work. In the referenced article, the hole doping level in graphene is controlled through the duration of exposure to oxygen plasma. Furthermore, in Ref. [25], surface plasmons in graphene are focused to produce an electromagnetic "hotspot" in a tapered waveguide at wavelength $1.55 \mu \mathrm{m}$ employing the charge transfer (CT) doping mechanism described in Section 7.A, specifically using the high work-function oxide $\mathrm{MoO}_{3}$. Section 7.B describes a potential fabrication method to obtain surfaces presenting the high spatial frequencies required to form the aforementioned phase-matching coupler (periods of the order of $10 \mathrm{~nm}$ ), while in Section 7.C, we explore the possibility of dilating the phase-matching coupler period to render it compatible with existing nanofabrication tools.

(a)

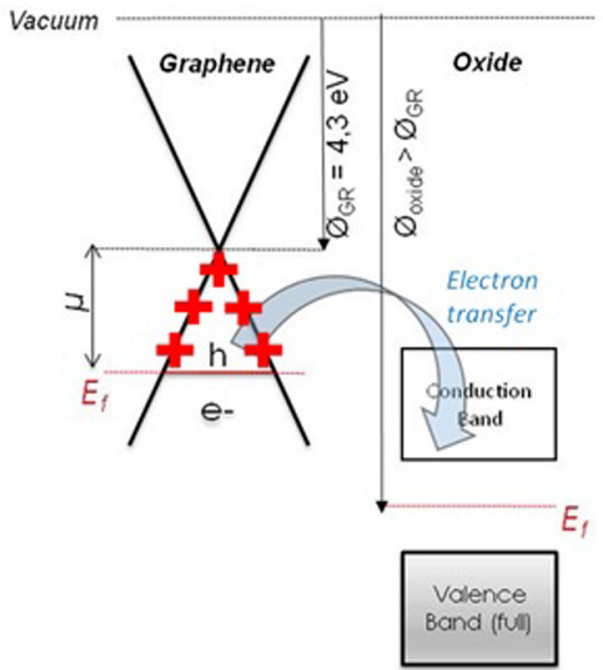

(c)

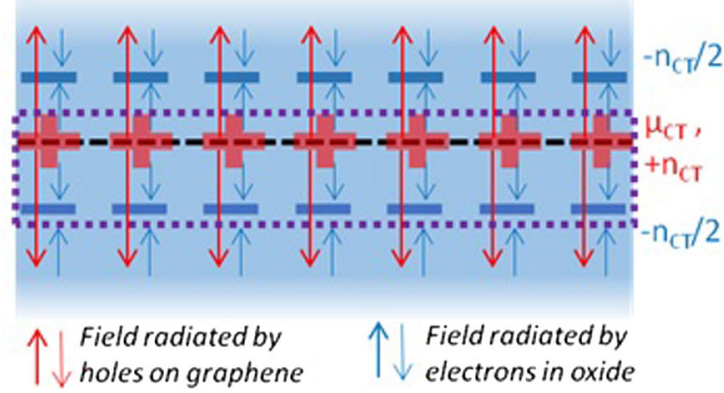

\section{A. Doping of Graphene with High Work-Function Oxides}

It is proposed that static doping of graphene via CT mechanisms to high work-function oxides be employed in tandem with electrical doping in order to reach the required doping levels mentioned previously. In the following section, this tandem operation will be described and shown to lead to electric fields over the thickness of the $10 \mathrm{~nm}$ oxide layer separating the graphene ribbons from the metal substrate.

Recent reports in the literature have shown that graphene can be doped through the transfer of charges to metal oxide materials presenting high work-functions, and thus unoccupied levels in their conductance bands that are situated energetically below the Dirac point of graphene [26-28]. Electrons from graphene can therefore be transferred to the oxide, thereby leaving the graphene with a p-type doping associated with holes in its valence band, as schematized in Fig. 8(a). In the proposed coupler device, the low-index material forming the slot region surrounding the graphene can be formed from such an oxide. In the literature, examples exist of CT doping of graphene up to $0.8 \mathrm{eV}$ using $\mathrm{MoO}_{3}$ as the high work-function oxide [25]. Upon contact of graphene with such an oxide, upon the hypothesis of a "sandwich" structure (i.e., charge extraction is possible on both sides of the graphene sheet), half of the extracted electrons

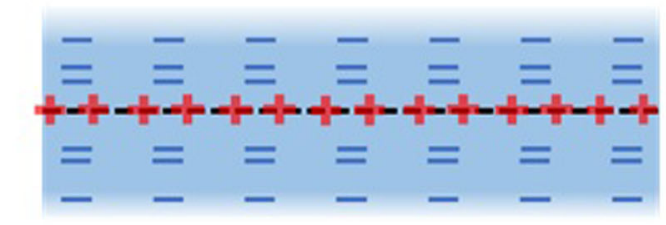

hole, - electron, oxide, ---graphene

(d)

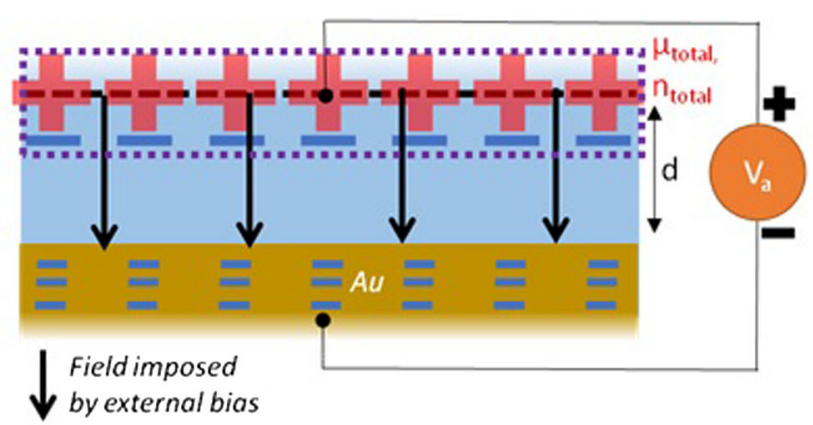

Fig. 8. (a) Schematic interpretation of the CT mechanism between graphene and high work-function oxides; (b) electrons transferred from graphene to oxide, leading to the formation of a space-charge region above and below the graphene; (c) approximation of space-charge regions to planes of charge of density $+n_{\mathrm{CT}}$ (holes on graphene), $-n_{\mathrm{CT}} / 2$ (electrons in oxide); cancellation of opposing radiated fields; chemical potential of graphene in the absence of external bias $=\mu_{\mathrm{CT}}$; (d) only the field imposed by the applied bias voltage acts upon the oxide in the capacitor structure. Chemical potential of graphene under external bias voltage $V_{a}=\mu_{\text {total }}$, for a surface charge density $n_{\text {total }}$. 
will be accepted by the oxide above the graphene and half by that below and are supposed to occupy a negligibly thin surface layer in both cases. The graphene, now at a chemical potential $\mu_{\mathrm{CT}}$ (indicating that induced by $\mathrm{CT}$ ) represents a plane with a surface charge density of $+n_{\mathrm{CT}}$, while the electrons accumulated in planes on either side of the graphene possess a density $-n_{\mathrm{CT}} / 2$, as shown in Fig. 8(c). The field radiated into the oxide thickness above and below the graphene is therefore zero, due to the cancellation of the fields emanating from the aforementioned charge planes.

We seek to show here that, having statically doped the graphene with a high work-function oxide, the electric field imposed by the application of an external bias in order to complement this initial doping and achieve chemical potentials of $\mu=1 \mathrm{eV}$ would not exceed the breakdown field of the oxide when employed in the aforementioned capacitor configuration. As stated in Section 5, the electrical gating involves applying a voltage $V_{a}$ over the lower oxide region of thickness $d=10 \mathrm{~nm}$ between the graphene ribbons and the $\mathrm{Au}$ substrate, as shown in Fig. 8(d). The induced electric field is
$E_{\text {gate }}=n_{\text {gate }} \cdot q_{e} /\left(\varepsilon_{0} \cdot \varepsilon_{d}\right)$, where the symbols take their previously ascribed meanings. In contrast to the previous calculation given in Section 5, we now assume that the chemical potential of graphene is nonzero for $0 \mathrm{~V}$ external bias voltage, but is rather $\mu_{\mathrm{CT}}$ (corresponding to a charge density of $n_{\mathrm{CT}}$ ). In order to find the extra surface charge density $n_{\text {gate }}$ needed to raise the chemical potential of graphene from $\mu_{\mathrm{CT}}$ to $\mu_{\text {total }}(=1 \mathrm{eV})$, we integrate over the density of states between these limiting chemical potential values,

$$
n_{\text {gate }}=\int_{\mu_{\mathrm{CT}}}^{\mu_{\text {total }}} \operatorname{DOS}(E) \mathrm{d} E=\int_{\mu_{\mathrm{CT}}}^{\mu_{\text {total }}} \frac{2|E|}{\pi \bar{h}^{2} v_{f}^{2}} \mathrm{~d} E=\frac{\mu_{\text {total }}^{2}-\mu_{\mathrm{CT}}^{2}}{\pi \bar{h}^{2} v_{f}^{2}},
$$

where $\operatorname{DOS}(E)$ is the areal density of states in graphene, and other symbols take their preascribed meanings. The calculated field is $E_{\text {gate }}=530 \mathrm{~V} / \mu \mathrm{m}$, this being of the same order of magnitude as the breakdown field of common oxides, e.g., $\mathrm{SiO}_{2}$ (we note that the exact breakdown voltage of the high workfunction oxides to be employed are unknown, but are supposed to be comparable). The required bias voltage can be calculated (a)
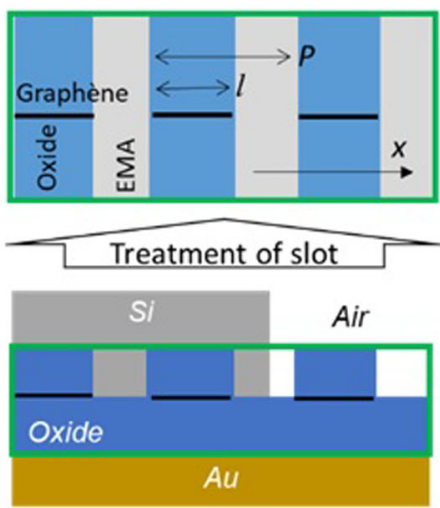

(b)
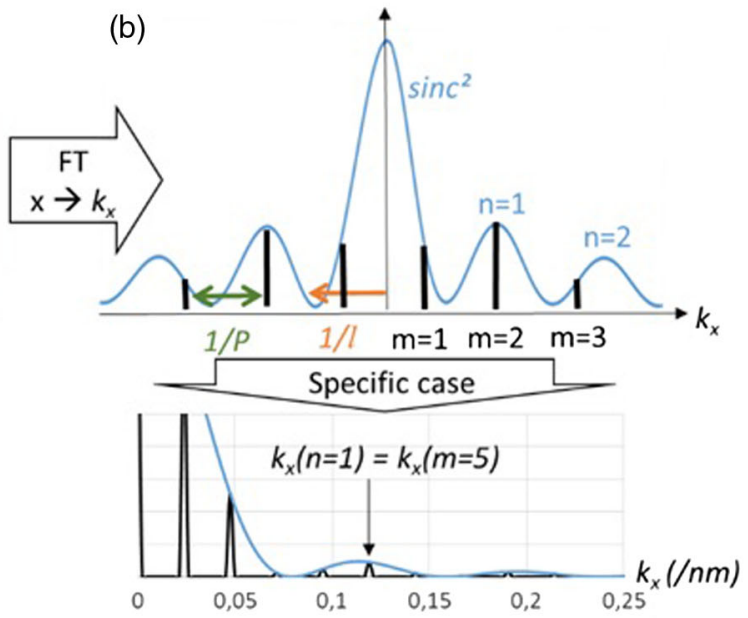

Free Space Wavelength $(\mu \mathrm{m})$

(c)

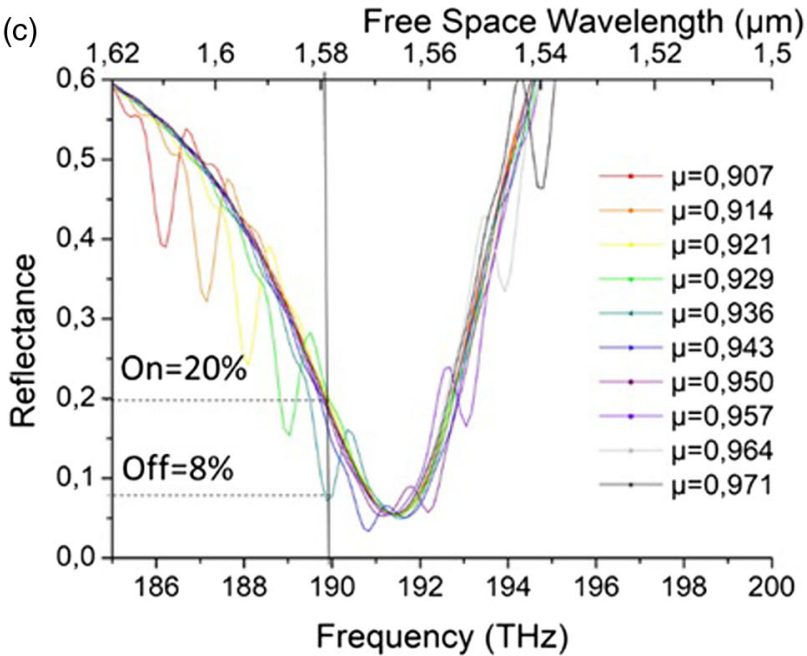

Fig. 9. (a) Simplified treatment of slot region featuring phase-matching coupler; (b) FT of the periodic structure of the slot region; top = schematized, general case showing the diffracted orders $m$ (whose spatial frequencies are shown by the black peaks) and the envelope sinc ${ }^{2}$ function linked to the length $l$ of the oxide teeth and graphene ribbons; bottom = specific case exploited in this work, where energy is channeled towards the fifth diffracted order $(m=5)$, where the diffracted orders are the black Dirac-like peaks; (c) reflectance spectra for various chemical potentials, showing a modulation between $8 \%$ and $20 \%$ reflectance when shifting between $\mu=0.95$ and $0.957 \mathrm{eV}$. 
as $V_{a}=E_{\text {gate }} \cdot d=5.3 \mathrm{~V}$. Finally, using the same method as described in Section 5 but substituting the required bias voltages for those allowing for a static doping with a high work-function oxide (namely, $V_{a}=5.6 \mathrm{~V}$ for $\mu=1.01 \mathrm{eV}$ and $V_{a}=5.9 \mathrm{~V}$ for $\mu=1.02 \mathrm{eV}$ ), the energy per bit could be reduced by an order of magnitude to $0.69 \mathrm{pJ} / \mathrm{bit}$.

\section{B. Fabrication of High Spatial Frequency Phase-Matching Couplers}

We propose the use of vicinal miscut substrates, coupled with step-bunching techniques to achieve a surface organization at the desired length scale for the phase-matching coupler. Reports in the literature [29] have demonstrated the fabrication of GaAs surfaces featuring $1 \mathrm{D}$ periodic structuration with lateral periods under $10 \mathrm{~nm}$ and amplitudes of around $5 \mathrm{~nm}$, which have typically been developed as substrates for the horizontal growth of nanowires. Beginning with a (311) GaAs substrate, monolayers of GaAs are grown via molecular beam epitaxy, followed by a high-temperature annealing step to allow surface reorganization. During the annealing, the deposited atoms migrate across the surface until they meet the edge of a terrace formed during the miscutting of the substrate and distribute themselves evenly along it. Furthermore, several terraces can merge, in a phenomenon known as step bunching, to form superstructures for which the height and length are integer multiples of the basic reticular plane separations. Such substrates, potentially conformally coated with a thin layer of the low-index oxide described previously, could provide the fine corrugation necessary for assuring phase matching with graphene's SPP.

\section{Use of Electron-Beam Lithography-Compatible Phase-Matching Gratings}

The phase-matching grating proposed previously requires state-of-the art electron-beam lithography tools in order to produce the fine features required (period $=16 \mathrm{~nm}$, oxide-teeth width $=8 \mathrm{~nm}$ ). While equipment capable of producing such features exists [30], we propose succinctly in this section the use of phase-matching gratings with lower spatial frequencies (higher periods) than discussed previously, rendering them compatible with more commonplace electron-beam lithography fabrication techniques. We note that common electron-beam systems are capable of delivering lateral resolutions under $50 \mathrm{~nm}$ when suitably thin layers of resist are employed [31,32], thereby preventing scattering of electrons in the thickness of the resist. In the case of the proposed device, the resist thickness need only be of the order of a few tens of nanometers, so as to allow the transfer of the pattern into the oxide-graphene-oxide sandwich (composing the slot layer of the proposed dual coupler system). With this degree of flexibility concerning the lateral structuration in mind, we propose enlarging the period of the phase-matching grating by a factor of 2.5 , i.e., from $16 \mathrm{~nm}$ up to $42 \mathrm{~nm}$, while the length of the oxide teeth (and the graphene ribbons) is increased by a factor of 1.5 , from $8 \mathrm{~nm}$ to $12 \mathrm{~nm}$. These enlargement factors are chosen (a) in the case of the oxide teeth to ensure that the graphene ribbons still host an integer number of half-wavelengths of the SPP so that there is constructive interference on a round trip, and (b) for the overall period such that the Fourier transform (FT) of a unit cell of the phasematching grating allows for an optimal channeling of energy into the (evanescent) diffracted order whose spatial frequency matches that of graphene's SPP, which is in this case the fifth order $(m=5)$. These criteria are schematized in Figs. 9(a) and 9(b). The reflectance spectra in Fig. 9(c) for various values of graphene's chemical potential show that, as expected, the coupling between the metal SPP and the graphene's SPP is clearly reduced. However, sufficient optical power is still exchanged to allow for a modulation contrast of $-4 \mathrm{~dB}$ near $1.55 \mu \mathrm{m}$ when the chemical potential is shifted by $7 \mathrm{meV}$.

\section{CONCLUSION}

A modulator device exploiting graphene's SPP communicating with free space via the intermediary of a metal SPP has been proposed and modeled using CMT and FDTD simulations. The dual-resonator system displays a frustration phenomenon whereby a narrow, frequency-tunable reflectivity peak is superimposed into the absorption dip of the metal SPP resonance, and the former can be displaced spectrally through the application of an external bias used to vary graphene's Fermi level. A possible application for such a coupler would be in the role of an electro-optical modulator device operating in free space in the telecommunications band, for which the reflectance can be varied between $6 \%$ and $87 \%$, and the power consumption of such a device is estimated to be $20 \%$ lower than that of conventional graphene-based modulators that seek to attenuate optical signals through absorption in graphene itself. The coupled-mode theory simulation results demonstrate that light incident on the device from free space can be coupled to graphene's SPP with an efficiency defined by the degree of coupling between the two SPP resonators with a maximum efficiency of $50 \%$, which is an interesting result in itself. Indeed, efficient coupling to graphene's SPP in the NIR to MIR spectral range would open up a host of applications, for example, in the chemical detection domain.

Funding. European Research Council H2020 (648546).

Acknowledgment. This project has received funding from the European Research Council (ERC) under the European Union's Horizon 2020 research and innovation programme (grant agreement $\mathrm{N}^{\circ} 648546$ GRAPHICS).

Disclosures. The authors declare no conflicts of interest.

\section{REFERENCES}

1. F. Bonaccorso, Z. Sun, T. Hasan, and A. C. Ferrari, "Graphene photonics and optoelectronics," Nat. Photonics 4, 611-622 (2010).

2. M. Liu, X. Yin, E. Ulin-Avila, B. Geng, T. Zentgraf, L. Ju, F. Wang, and $X$. Zhang, "A graphene-based broadband optical modulator," Nature 474, 64-67 (2011)

3. Z. Sun, A. Martinez, and F. Wang, "Optical modulators with $2 \mathrm{D}$ layered materials," Nat. Photonics 10, 227-238 (2016).

4. V. Sorianello, M. Midrio, G. Contestabile, I. Asselberghs, J. Van Campenhout, C. Huyghebaert, I. Goykhman, A. K. Ott, A. C. Ferrari, and M. Romagnoli, "Graphene-silicon phase modulators with gigahertz bandwidth," Nat. Photonics 12, 40-44 (2018). 
5. O. Balci, E. Polat, N. Kakenov, and C. Kocabas, "Graphene-enabled electrically switchable radar-absorbing surfaces," Nat. Commun. $\mathbf{6}$, 6628 (2015).

6. H. Li, Y. Anugrah, S. Koestera, and M. Li, "Optical absorption in graphene integrated on silicon waveguides, Appl. Phys. Lett. 101, 111110 (2012).

7. Q. Guo, C. Li, B. Deng, S. Yuan, F. Guinea, and F. Xia, "Infrared nanophotonics based on graphene plasmonics," ACS Photon. 4 , 2989-2999 (2017).

8. M. Furchi, A. Urich, A. Pospischil, G. Lilley, K. Unterrainer, H. Detz, P. Klang, A. M. Andrews, W. Schrenk, and G. Strasser, "Microcavityintegrated graphene photodetector," Nano Lett. 12, 2773-2777 (2012).

9. X. Gan, R. J. Shiue, Y. Gao, K. F. Mak, X. Yao, L. Li, A. Szep, D. Walker, Jr., J. Hone, T. F. Heinz, and D. Englund, "High-contrast electrooptic modulation of a photonic crystal nanocavity by electrical gating of graphene," Nano Lett. 13, 691-696 (2013).

10. C. C. Guo, Z. H. Zhu, X. D. Yuan, W. M. Ye, K. Liu, J. F. Zhang, W. $\mathrm{Xu}$, and S. Q. Qin, "Experimental demonstration of total absorption over $99 \%$ in the near infrared for monolayer-graphene-based subwavelength structures," Adv. Opt. Mater. 4, 1955-1960 (2016).

11. Y. S. Fan, C. C. Guo, Z. H. Zhu, W. Xu, F. Wu, X. D. Yuan, and S. Q. Qin, "Monolayer-graphene-based perfect absorption structures in the near infrared," Opt. Express 25, 13079-13086 (2017).

12. H. Cheng, S. Chen, P. Yu, X. Duan, B. Xie, and J. Tian, "Dynamically tunable plasmonically induced transparency in periodically patterned graphene nanostrips," Appl. Phys. Lett. 103, 203112 (2013).

13. S. X. Xia, X. Zhai, Y. Huang, J. Q. Liu, L. L. Wang, and S. C. Wen, "Graphene surface plasmons with dielectric metasurfaces, J. Lightwave Technol. 35, 4553-4558 (2017).

14. S. Vantasin, Y. Tanaka, and T. Shimura, "Launching and control of graphene plasmons by nanoridge structures," ACS Photon. 5, 1050-1057 (2018).

15. Z. Fang, Y. Wang, A. E. Schlather, Z. Liu, P. M. Ajayan, F. J. Garcia de Abajo, P. Nordlander, X. Zhu, and N. J. Halas, "Active tunable absorption enhancement with graphene nanodisk arrays," Nano Lett. 14, 299-304 (2013).

16. S. Kim, M. S. Jang, V. W. Brar, K. W. Mauser, L. Kim, and H. A. Atwater, "Electronically tunable perfect absorption in graphene," Nano Lett. 18, 971-979 (2018).

17. Z. Wang, T. Li, K. Almdal, N. A. Mortensen, S. Xiao, and S. Ndoni, "Experimental demonstration of graphene plasmons working close to the near-infrared window," Opt. Lett. 41, 5345-5348 (2016).

18. X. Zhao, L. Zhu, C. Yuan, and J. Yao, "Tunable plasmon-induced transparency in a grating-coupled double-layer graphene hybrid system at far-infrared frequencies," Opt. Lett. 41, 5470-5473 (2016).

19. B. Wei, H. Liu, G. Ren, Y. Yang, S. Ye, L. Pei, and S. Jian, "Graphene based silicon-air grating structure to realize electromagneticallyinduced-transparency and slow light effect," Phys. Lett. A $\mathbf{3 8 1}$ 160-165 (2017).
20. X. Zhu, W. Yan, P. U. Jepsen, O. Hansen, N. A. Mortensen, and S. Xiao, "Experimental observation of plasmons in a graphene monolayer resting on a two-dimensional subwavelength silicon grating," Appl. Phys. Lett. 102, 131101 (2013).

21. R. Kundu, "Tight binding parameters for graphene," Mod. Phys. Lett. B 25, 163-173 (2011).

22. D. Legrand, L. Le Cunff, A. Bruyant, R. Salas-Montiel, Z. Liu, B. Tay, T. Maurer, and R. Bachelot, "Surface plasmons in suspended graphene: launching with in-plane gold nanoantenna and propagation properties," Opt. Express 25, 17306-17321 (2017).

23. T. Sun, J. Kim, J. M. Yuk, A. Zettl, F. Wang, and C. Chang-Hasnain, "Surface-normal electro-optic spatial light modulator using graphene integrated on a high-contrast grating resonator," Opt. Express 24, 26035-26043 (2016).

24. E. Timurdogan, C. Sorace-Agaskar, J. Sun, E. S. Hosseini, A. Biberman, and M. Watts, "An ultralow power athermal silicon modulator," Nat. Commun. 5, 4008 (2014).

25. Q. Zhang, X. Li, M. M. Hossain, Y. Xue, J. Zhang, J. Song, J. Liu, M. Turner, S. Fan, Q. Bao, and M. Gu, "Graphene surface plasmons at the near-infrared optical regime," Sci. Rep. 4, 6559 (2015).

26. Z. Chen, I. Santoso, R. Wang, L. F. Xie, H. Y. Mao, H. Huang, Y. Z. Wang, X. Y. Gao, Z. K. Chen, D. Ma, A. T. S. Wee, and W. Chen, "Surface transfer hole doping of epitaxial graphene using thin film," Appl. Phys. Lett. 96, 213104 (2010).

27. J. Meyer, P. R. Kidambi, B. C. Bayer, C. Weijtens, A. Kuhn, A Centeno, A. Pesquera, A. Zurutuza, J. Robertson, and S. Hofmann, "Metal oxide induced charge transfer doping and band alignment of graphene electrodes for efficient organic light emitting diodes," Sci. Rep. 4, 5380 (2014).

28. Q. Zhang, X. Li, M. M. Hossain, Y. Xue, J. Zhang, J. Song, J. Liu, M. D. Turner, S. Fan, Q. Bao, and M. Gu, "Graphene surface plasmons at the near-infrared optical regime," Sci. Rep. 4, 6559 (2014).

29. V. R. Yazdanpanah, Z. M. Wang, and G. J. Salamo, "Highly anisotropic morphologies of $\mathrm{GaAs}(331)$ surfaces," Appl. Phys. Lett. 82, 1766-1768 (2003).

30. H. Hiroshima, S. Okayama, M. Ogura, and M. Komuro, "Nanobeam process system: an ultrahigh vacuum electron beam lithography system with $3 \mathrm{~nm}$ probe size," J. Vac. Sci. Technol. B 13, 2514-2517 (1995).

31. H. Duan, "Sub-10-nm half-pitch electron-beam lithography by using poly (methyl methacrylate) as a negative resist," J. Vac. Sci. Technol. B 28, C6C58-C6C62 (2010).

32. V. Manfrinato, L. Zhang, D. Su, H. Duan, R. Hobbs, E. Stach, and K. Berggren, "Resolution limits of electron-beam lithography toward the atomic scale," Nano Lett. 13, 1555-1558 (2013). 